\title{
John Williamson and the terrifying results of the Medical Practice Information Demonstration Project
}

\section{Neuhauser}

John W Williamson, MD, was born in 1931 in Salt Lake City. He gained a BA degree in 1953 from the University of California, Berkeley and an MD degree in 1956 from the University of California, San Francisco. After residency in internal medicine he did a fellowship at the Office of Research in Medical Education at the University of Illinois in Chicago from 1963 to 1965 . From 1966 to 1984 he was on the faculty of the School of Public Health at Johns Hopkins University. From 1984 to 1999 he held senior administrative positions in research and education at the Veterans Affairs Medical Center in Salt Lake City. He is now retired and living in San Diego. In 2000 he was given the individual Ernest Amory Codman award of the Joint Commission on Accreditation of Healthcare Organizations for his life time of scholarship and other contributions related to quality of health care.

Here is how Avedis Donabedian described John Williamson's contributions in a letter written in July 2000. "Many years ago, much before the assessment of outcomes became so fashionable a slogan, Williamson perceived that 'outcomes' should indeed be the focus of attention in assessing the quality of care ..." Williamson's concept of quality is "achievable benefit not achieved". Donabedian said that Williamson's 1978 book "Assessing and improving health care outcomes" "deserves to be one of the classics in our field". "Williamson insisted that assessment of quality should involve not only physicians but a team of health workers cognizant of the issues at hand. I should add that Williamson can be considered the founder of a school of quality studies", influencing the work of Robert Brook, Evert Reerink and Alison Kitson.*

John is now retired and living in San Diego. He is ready to talk and laugh and seems more bemused than angry over the consequence of his 1976-9 study of the evidence in support of quality of care. How could such a person get into trouble in pursuit of better quality?

\section{THE NEED FOR EVIDENCE ABOUT BEST PRACTICE}

In 1975 William Munier, MD was the national director of the Professional Standards Review Organizations (PSROs) working for Theodore Cooper, MD who was Assistant Secretary for Health in the US Department of Health Education and Welfare (HEW) from 1975 to $1977 .{ }^{23}$ The PSROs were created at the state level of government to review the use of services and quality of care related to the Federal Medicare program. ${ }^{45}$

*Other books by Williamson include "Improving medical practice and health care. A bibliographic guide to information management in quality assurance and continuing education", Cambridge, Mass: Ballinger, 1977, 1035 pp (containing 3500 abstracts) and Williamson JW, Weir CR, Turner CW, et al. "Health care informatics and information synthesis", Thousand Oaks, CA: Sage, 2002.

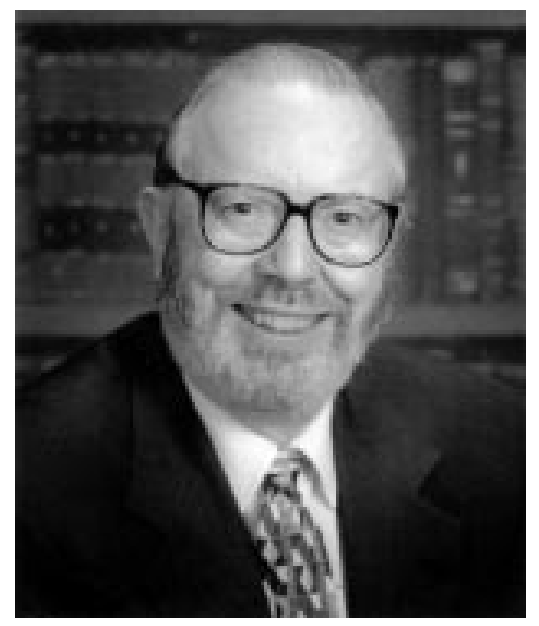

PSRO staff did examine length of hospital stays, but were mostly mystified over how to set standards for quality of care evaluation. Williamson and his colleague Peter Goldschmidt, MD (publisher of "Report on medical guidelines and outcomes research medical care management corporation" and co-author of "Quality management in health care") proposed to Munier that this gap might be addressed by inviting leading experts to assess the state of knowledge about diseases and their treatment. The experts would be chosen by the appropriate branch of the National Institutes of Health (NIH). These experts would be asked specific questions about outcomes of care and to support their answers with the best available evidence. Thus, best practices and quality could be defined and the conclusions used to guide PSROs in evaluating quality.

\section{WILLIAMSON'S STUDY}

Munier took this idea to Cooper who told him it was very "important". Cooper asked Munier to talk about it with Donald Fredrickson, MD, the distinguished head of NIH from 1975 to 1981. Munier recalls Fredrickson being very polite at their meeting. Later Fredrickson and Cooper talked about it by telephone. Munier, many years later, recalls Cooper's description of the conversation. Cooper said Fredrickson was not in favour of the proposal. Cooper told Fredrickson he had six months to come up with his own plan if he did not like this one. He did not and in 1976 Williamson, along with Peter Goldschmidt and Irene Jillson, were given a contract through their company, Policy Research Incorporated, to carry out the Medical Practice Information Demonstration Project (MPIDP). ${ }^{6}$ Susan Horn, $\mathrm{PhD}$, and Theodore Colton, $\mathrm{PhD}$, helped to organize a panel of leading biostatisticians to assess the quality of the literature. ${ }^{7}$ Munier was the government director for the project and he contacted the heads of several NIH institutes to invite them to participate. Those who agreed were asked to select a health condition within their domain where research had contributed 
substantially to improving patient outcomes. The National Cancer Institute chose malignant melanoma, the National Heart Lung and Blood Institute picked chronic rheumatic heart disease, and the National Institute of Mental Health chose bipolar disorder. Each of these institutes selected 20-40 experts across the country to help evaluate the evidence related to care. Experts were chosen in the areas of epidemiology, diagnosis, treatment and economics. To guide the decision making, Williamson's group developed workbooks with specific questions to be answered such as: incidence and prevalence of the condition, diagnostic test sensitivity and specificity, treatment efficacy (under ideal conditions) and effectiveness (in usual clinical practice), and economic costs to the nation. Critical information being sought included:

- the magnitude of the problem, incidence, prevalence, natural history (without health care) and social (including economic) costs;

- achievable benefits from current health care technology if optimally applied;

- extent of achievable benefits actually being achieved at present;

- cost effectiveness of reasonable improvement action (Williamson J, Goldschmidt P, Jillson I, et al. "The Medical Practice Information Demonstration Project". January 1984, unpublished, page 5).

The literature was searched by finding all citations for the three topics listed by Medline, the main computerized bibliography of the National Library of Medicine (NLM). This yielded 2696 citations. A random sample of 365 of these were drawn and analyzed independently by two biostatisticians, at least one of whom was a specialist on the topic. Of these, 37\% were "conceivably applicable in some way to practice" and only $4 \%$ "met criteria of information maturity for either quality assurance or clinical practice." $\$+$ Workbooks containing the best evidence were sent to the expert panel. These experts were asked to provide the best available estimates for data points such as: the expected health outcome for a 25 year old white woman with stage I malignant melanoma of the head or neck, given current treatment. The experts were asked to provide the research citations for the best documentation of their answer, and whether their answer was based on research, extrapolation, or assumption.

\section{THE RESULTS OF THEIR RESEARCH}

Across all three topics a total of 872 such data points were requested. Only 130 (15\%) were supported by a direct research study. The statistical team for the overall study found that, of these 130, only 30 of the data points were actually contained in the cited study. Only six of the 872 data points were "even moderately substantiated by empirical research". 298 requests for best articles were asked of the experts. For 245 at least one expert said there was "no valid data or acceptable article".

According to Williamson: "On reading the comments provided by the expert teams in response to our post-study project evaluation questionnaire, the following comments were typical of their reactions. One stated that it was depressing to see that the state of the art is so primitive. Another stated that it is frightening to think that such uncertain material can serve as the basis of policy; indeed terrifying."

Apart from the soundness of the information, Williamson found serious problems retrieving the relevant research information. For example, the experts in bipolar disease listed 124

†This paper briefly summarizes Williamson's MPIDP project and was in print before the embargo was imposed (Williamson, personal communication, February 2002). key articles. When their list was compared with the 1640 articles on bipolar disorder in Medline only 13 overlapped. The National Institute of Mental Health bibliographic file had 294 articles on this topic of which only eight overlapped with the experts' list. "Only a single article could be referenced through all three sources." ${ }^{8}$ The leading textbook (by team consensus) for bipolar disorder had 859 citations, only 46 of which could be found on Medline. In short, there was no common body of literature on best practice-another worrisome finding.

According to Goldschmidt, Munier and Williamson, long term senior staff at NIH were not happy about this project. This negative reaction is understandable since the project was imposed on NIH by the Assistant Secretary for Health. The findings posed a potential political threat to the NIH because it justified its government funding based on improvement of care. The NLM was supposed to be giving practitioners the published evidence they needed to practice appropriately. Dr Martin Cummings, Director of this Library and a recipient of multiple honorary degrees, ${ }^{9}$ wrote a letter to the powerful US Representative Henry Waxman attacking Williamson's study.

By that time key government backers of the study were gone. On 20 January 1977 at 12 noon Ted Cooper was relieved of his position by the new Secretary of HEW, Joseph Califano. Afterwards Dr Munier said he was "cornered by the Carter people" and left government service two years later.

The contract came to an end in 1979 with a report to the Assistant Secretary of Health. NIH staff took the very unusual step of retrospectively peer reviewing Williamson's report. These peers were critical and said "This research is leading to no useful result". Williamson was told that he could publish no reports from his study. Munier thinks that from then on Williamson was blackballed by the NIH. Today Williamson says he was told, off the record, that future grant proposals from him to the NIH would not be looked upon favorably. $\neq$ His report remains unpublished to this day.

Williamson spent the years 1981-1983 as a Visiting Professor in the Department of Biostatistics at Harvard University's School of Public Health. He resigned his Johns Hopkins Professorship in 1984 to take a senior position at the Veterans Administration Medical Center in Salt Lake City, the place of his birth, where he continued his research on quality of care. Today Munier thinks that the trouble at NIH led to Williamson's move to Salt Lake City. According to Horn: "I can remember the pain it caused him. He always wanted to be discrete about the situation ... He was a true visionary, but few wanted to listen" (S Horn, personal communication, 8 April 2002). Today Munier (personal communication, 5 April 2002) thinks that "so much could have been accomplished if the report had been received in an intellectually honest way and acted upon". He thinks that this study influenced the later work of the NIH Office of Medical Application of Research (OMAR) which, since the 1980s, has produced a long series of consensus panel reports on best medical practice. A later director of the NLM, Dr Donald Lundberg, put much effort into practitioner friendly computerized bibliographies. The US National Clearinghouse of Practice Guidelines has been established.

With the benefit of 25 years of hindsight, Williamson's findings are not surprising. The evidence based medicine movement is working to fill in this lack of evidence. Today the Cochrane Collaboration has collected information from 250000 randomized clinical trials, ${ }^{10}$ and we could use many more. NIH and NLM continue to prosper and are highly

†There is no written documentation in support of Munier and Williamson's perception. Such prejudgement would be completely inconsistent with official NIH policy (Williamson, personal communication 30 July 2002). Note, however, that Williamson's following career moves can be understood by this perception of his. 
regarded. In short, we now realize that John Williamson and his colleagues were right in 1979, but some were not ready for his message then. Transparency and an honest willingness to evaluate what we do and know are worthy goals. They will be essential to our efforts to reduce error and improve quality.

\section{Author's affiliation}

D Neuhauser, The Charles Elton Blanchard Professor of Health Management, Professor of Epidemiology and Biostatistics, Co-Director, Health Systems Management Center, Case Western Reserve University, Cleveland, OH 44106-4945, USA; dvn@po.cwru.edu

\section{REFERENCES}

1 Williamson J. Assessing and improving health care outcomes. Cambridge, Mass: Ballinger, 1978.
2 Who's who in America 1983-1984.43rd ed. New Providence, NJ: Marquis Who's Who.

3 Who's who in medicine and health care 1997-1998. 1st ed. New Providence, NJ: Marquis Who's Who.

4 Decker B, Bonner P. PSRO: organization for regional peer review. Foreword by Paul Batalden. Cambridge, Mass: Ballinger, 1973.

5 Bussman J, Davidson S. PSRO: the promise, perspective and potential. Menlo Park, CA: Addison-Wesley, 1981.

6 USHEW. Contract 282-77-0068GS. Medical Practice Information Demonstration Project. Final Report. 1979.

7 Horn SD, Williamson JW. Statistical analysis of the reliability and validity of nominal group priority judgements in health care. Med Care $1977 ; 15: 922-8$.

8 Williamson JW. Information management in quality assurance. Nursing Res 1980:29:78-82.

9 Who's who in health care. 2nd ed. Rockville, MD: Aspen, 1981: 101-2. 10 The Economist 2 March 2002, page 74.

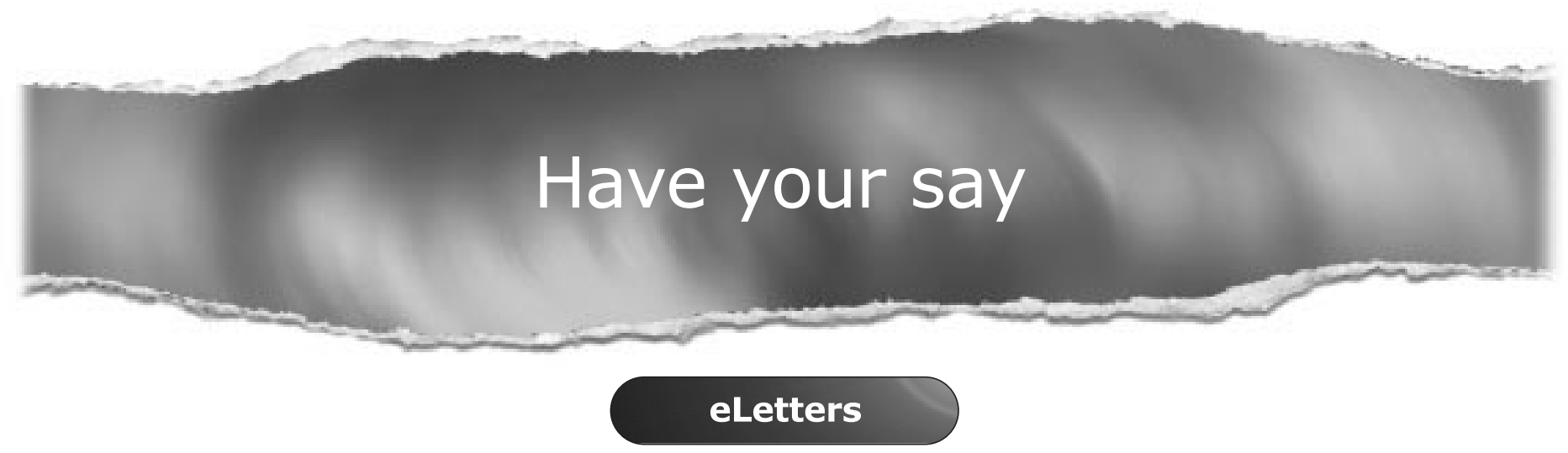

If you wish to comment on any article published in Quality and Safety in Health Care you can send an eLetter using the eLetters link at the beginning of each article. Your response will be posted on Quality and Safety in Health Care online within a few days of receipt (subject to editorial screening).

\section{www.qualityhealthcare.com}




\section{ORGANISATIONAL MATTERS}

\section{Healthcare redesign: meaning, origins and application}

L Locock

Qual Saf Health Care 2003;12:53-58

Healthcare organisations are using redesign to tackle variation in the quality of care and improve public satisfaction. It is represented as a radical challenge to traditional assumptions and practices which involves thinking through the best process to achieve speedy and effective patient care, identifying delays, unnecessary steps, or potential for error, and redesigning the process to improve the quality of care. This paper explores the meaning of redesign using practical illustrations. It examines its theoretical origins, particularly total quality management (TQM)/continuous quality improvement (CQI) and re-engineering, and assesses evidence which may inform its application. This evidence suggests that clinical ownership and senior management support will be essential. Redesign seeks to balance the more gradual approach of TQM with the organisation-wide lateral thinking of re-engineering. An incremental negotiated approach seems more likely to ensure clinical ownership, but carries a risk that $Q$ I will remain small scale with little impact on the wider organisation. Inclusion of some re-engineering techniques may help to overcome this difficulty. Evidence suggests that most Ql techniques achieve only partial success. This may pose difficulties for redesign, which has generated high political expectations that it can solve long term problems in health care.

Correspondence to: L Locock, Department of Social Policy and Social Work, University of Oxford, Barnett House, Wellington Square, Oxford OXI 2ER, UK;

louise.locock@socres.ox.ac.uk
R edesigning healthcare systems has become an international preoccupation, as policy makers seek new ways to address continuing problems of variation in the quality of health care and dissatisfaction among patients, the public, and professionals. As an editorial in the $B M J$ recently commented:

"Healthcare systems fail to provide treatments that are known to work, persist in using treatments that don't work, enforce delays, and tolerate high levels of error. Healthcare leaders are now recognising . . .that the healthcare system needs radically redesigning."

Many countries now have national healthcare quality improvement agencies which are using redesign techniques. Examples include the Institute of Healthcare Improvement (IHI) in the US (one of the foremost advocates of redesign), ${ }^{2}$ the Modernisation Agency in the UK National Health Service (NHS), ${ }^{3}$ the Dutch Institute for Healthcare Improvement (CBO) ${ }^{4}$ and the Australian Council for Safety and Quality in Healthcare. There is substantial international exchange of ideas and techniques between these various bodies, and the relationship between IHI and the NHS Modernisation Agency is particularly close. This international exchange could be seen as a form of collective policy "puzzling" as ideas are borrowed, adapted, tested, and reformulated.

Partly because of this debate, the concept of redesign is rapidly evolving and the word is used to describe a family of approaches rather than a single technique. However, what can be said is that it is not just another word for "change" or "reorganisation"; to redesign enthusiasts it conveys a specific set of meanings, and represents a radical challenge to healthcare providers to reconsider their whole approach to improving the quality of services.

As ever, the interest of policy makers in new initiatives needs to be understood within a wider political context. In the case of the UK, the Labour government has recently announced major increases in healthcare funding but, in return, expects fundamental reform. The Prime Minister's foreword to the NHS Plan of $2000^{7}$ describes this as offering the nation and those in the NHS a "deal":

"We would spend this money if, but only if, we also changed the chronic system failures of the NHS. Money had to be accompanied by modernisation; investment, by reform ... It is, in a very real sense, our chance to prove for my generation and that of my children, that a universal public service can deliver what people expect in today's world."

Although the British government has explicitly rejected the idea of moving from a tax funded system to one based on social insurance, the implication is that the NHS will find it difficult to defend itself against demands for changes in its funding base unless it can improve dramatically. In fact, the interest shown in redesign by other countries with completely different systems suggests the problems in meeting consumer expectations are less to do with the potential inefficiencies of public welfare provision than the collective power structures and traditional professional and managerial practice.

This paper explores the meaning of redesign as the term is currently used, illustrated with practical examples; examines the theoretical origins of redesign; and assesses available evidence which may inform its application. The objective is to explore how far redesign is in fact a distinctive new approach or a blend of existing techniques, and how likely it is to succeed.

\section{THE MEANING OF "REDESIGN"}

Healthcare redesign can be broadly defined as thinking through from scratch the best process to achieve speedy and effective care from a patient perspective, identifying where delays, unnecessary steps or potential for error are built into the 
process, and then redesigning the process to remove them and dramatically improve the quality of care.

In principle, healthcare organisations around the world have always aimed to serve patients. In practice, however, they do not always put patients' needs and preferences first over the convenience of the organisation. Established ways of doing things often remain unquestioned; the daily struggle to keep existing processes going with limited resources"hamster health care" as it has been aptly described-rarely allows space to step back and reflect critically. Redesign seeks to challenge this organisational treadmill and to question practices that are taken for granted.

This means not just doing more of the same, or doing it slightly differently, but rethinking the assumption that it needs to be done at all, and whether what is done at present adds any value to patients (or, indeed, to hard pressed staff). An important principle of redesign is that it should be led by frontline staff themselves, who are best placed to know what currently happens and develop creative ideas for improvement. Typical steps in a redesign initiative might include:

- Mapping the existing care process (sometimes described as the "as is" stage).

- Analysing where problems exist in that process and questioning why each step is done, by whom, where, in what sequence, and is there a better way?

- Imagining what an "ideal" process might look like (sometimes described in re-engineering as "visioning")

- Identifying practical changes to the current process to make it closer to the ideal process.

- Testing these changes and evaluating whether they result in improvement.

In the initial process mapping stage frontline staff of all disciplines connected with a process pool their different perspectives to write down the different steps patients go through and what delays they encounter. Individual staff may feel their own "piece of the jigsaw" is straightforward and efficient, but may never have seen how it fits into the wider picture and how the whole system could be made more efficient. The process map can be represented as a simple text list or as a formal flow chart using different symbols or shapes to represent steps such as "transport", "waiting", or "decision making". A simple illustration is given in fig 1 .

Ideally, patients should also be directly involved in process mapping. However, patients may not feel sufficiently empowered and staff may feel reluctant to explore flaws in the process honestly with patients present. Indirect means of patient involvement such as confidential interviews, patient diaries, and observational tracking of patients through the system can help to overcome this.

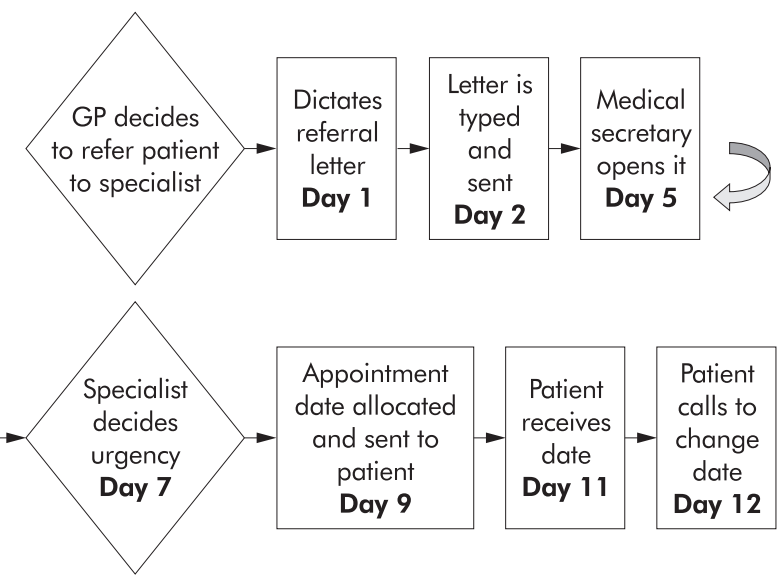

Figure 1 Process map.

\section{Box 1 Characteristics of TQM}

- Continuous incremental improvement of curren processes-repeated testing and evaluation of small scale changes.

- Responsibility for quality in the hands of frontline staff.

- Collective team responsibility.

- Detailed meticulous measurement

- Reporting of errors and defects without fear of blame.

- Culture of open questioning and constant learning.

- Organisation-wide philosophy of quality as everyone's business.

\section{Box 2 Characteristics of re-engineering}

- Radical transformational change of whole organisation simultaneously, abandoning current practice.

- Focus on rethinking and redesigning processes from scratch.

- Strip out all unnecessary steps.

- Led from the top down-emphasis on strong management control and visionary leadership.

- Decision making at the level where the work is carried out-team empowerment.

- Requirement for flexible work practices.

- Strong emphasis on IT.

\section{THEORETICAL ORIGINS OF REDESIGN}

Healthcare redesign draws on various quality improvement theories but seeks to incorporate learning from past experience of applying them to health care. These include, amongst others, the theory of constraints, ${ }^{9}$ lean thinking, ${ }^{10}$ and complexity theory. ${ }^{11}$ However, two particularly important strands of influence will be examined here-total quality management (TQM)/continuous quality improvement $(\mathrm{CQI})^{12-14}$ and re-engineering. ${ }^{15}$ Both approaches have a primary focus on the needs of the customer or user, not the needs of the organisation, and examine whole processes rather than single departments or tasks. However, they recommend strongly contrasting methods: the guiding philosophy of TQM is continuous incremental improvement, while re-engineering is based on radical rapid transformation. The key characteristics are summarised in boxes 1 and 2 .

In health care and beyond, both TQM/CQI and reengineering have promised substantial change, but the reality has rarely matched expectations. Evidence on the extent of change and factors which have affected implementation are explored below. (It should be noted that much of the literature in both fields is either prescriptive in nature or presents the experience of individual stakeholders and enthusiasts; independent research evidence is relatively scarce).

In principle, continuous quality improvement led by frontline staff would seem an appropriate choice for health care, given all the evidence that "top down" imposition of change rarely succeeds in getting autonomous clinical professionals to change practice. ${ }^{16}$ An approach led by clinicians which allows for gradual negotiation and implementation seems more likely to be accepted. It has also been suggested that the emphasis of TQM on systematic measurement and evaluation appeals to doctors' scientific training.

However, available evidence on TQM/CQI in health care suggests that improvements in quality have been slow to materialise and relatively small scale. CQI techniques may have some success within individual teams or departments, but changes made have tended to be limited in scope and impact. There is little evidence to support the claim that TQM programmes will act as a catalyst to achieve organisation-wide change. ${ }^{17-19}$ 
Box 3 Example 1: Reducing medication error and waste in the US

The Medical Center of Ocean County in New Jersey has redesigned its procedures for supplying drugs to the ward. The original process was the traditional "cart system" used by the majority of US hospitals: each evening the pharmacy collected medication orders for the following day, dispensed the medications, and placed them in carts to be delivered to nursing stations early next morning. Analysis of this system showed that, following ward rounds the next day, medications would often be changed but the drugs on the ward cart were still those based on the previous day's orders. This created extensive rework on the ward and in the pharmacy, and a high potential for confusion and error. Furthermore, in anticipation of problems arising, nursing staff would store additional supplies of several drugs on the ward, creating further waste and potential for mistakes.

The pharmacy studied the distribution of medication orders and found that $38 \%$ were scheduled to be administered at 09.00 hours. Smaller peaks were found at 13.00 17.00 , and 21.00 hours. On the basis of this information, a new "just-in-time" delivery system was piloted in which four delivery times were introduced at 08.00, 12.00, 16.00 , and 21.00 hours. To support the new system a pharmacy technician was assigned to each floor of the hospital for 16 hours per day. The technician evaluates medication orders, inputs them into the system, and liaises between clinical staff and the pharmacist. The new system has reduced error, improved availability of the correct drugs at the correct time, and reduced waste. ${ }^{33}$

Box 4 Example 2: Direct booking for cataract surgery in the UK

Peterborough Hospitals Trust has worked with community services to redesign its cataract service. Mapping of the existing process for referral for cataract surgery showed that delays were built into the process by requiring all referrals to be made by a GP to a consultant ophthalmologist, who would then assess the patient in the outpatient department before sending out a date for surgery. Visioning suggested that it would be more efficient for patients with obvious cataract to be booked directly onto an operating list, bypassing the need for a GP or outpatient appointment.

A group of optometrists have therefore been trained to assess and counsel patients for surgery in the community using agreed criteria. At the end of the consultation the optometrist books a day surgery slot directly with the hospital for a date agreed on the spot with the patient. Patients fill in a questionnaire on their general health and return it to the hospital for preoperative assessment. Nurses in the ophthalmology department check the questionnaire and telephone the patient to discuss any problems. The time from diagnosis to operation has been reduced from many months to 6 weeks; there are now no clinic appointments, fewer patient journeys, no duplication, and reduced costs. ${ }^{34}$

There are clear messages from these studies that, where some progress has been made, the engagement of clinicians has been essential, as well as senior management commitment and persistence. However, professional resistance to
Box 5 Example 3: "Breakthrough" collaboratives

A growing number of "collaboratives" 35 have been established in several countries to improve care across the whole patient journey. More than 700 teams from US and Canadian healthcare organisations and more than 800 project teams in the UK have participated in collaborative programmes, including (in the UK) primary care, cancer, orthopaedics, critical care, medicines management, coronary heart disease, and mental health. Collaboratives bring together networks of clinical and managerial staff from several healthcare organisations. They work together for several months on improving a specific clinical service, providing mutual support and shared learning. Collaboratives use an accelerated model for quality improvement developed in the US by Nolan and colleagues ${ }^{36}$ and adapted for use in healthcare by Don Berwick, Director of the IHI. The model uses the "Plan-Do-Study-Act" (PDSA) cycle which aims to test and implement ideas for improvement quickly and practically in real work settings, and uses the results to inform and guide further work. It starts with small changes which in theory can then be built into larger improvements through successive PDSA cycles.

Numerous service improvements achieved through collaboratives have been reported. A few examples from the UK cancer collaborative include the following:

- In mid-Anglia radiologists started to refer directly to the chest physician if they found signs of cancer which reduced waiting times from an average of 24 days to 11 .

- In Leicestershire those suspected of having bowel cancer used to have separate visits (and two bowel preparations) for flexible sigmoidoscopy, barium enema, and consultation; now they can do all three on one day.

- In Merseyside and Cheshire a prebooking system reduced the waiting time for bowel biopsy results from 5 weeks to 3 .

- In south east London the introduction of an assessment by a palliative care nurse meant that the delay in starting care was reduced from 2-4 weeks to a maximum of 2 days. ${ }^{37}$

what is seen as an imported business technique has been a common obstacle.

Pollitt ${ }^{20}$ has argued that many business approaches to quality improvement, including both TQM and re-engineering, have failed to take account of the complexity of health care and the nature of professionalised knowledge. The language and values used are alien to health care and are rejected as management fads.

Re-engineering was a response to the perceived failures of TQM's incrementalism and failure to achieve organisationwide change but, as with TQM, research suggests that the gains made in health care (as in other organisations) have not been as great as predicted. ${ }^{21-24}$ A particular problem has been its aggressive rhetoric and its failure to engage the staff on whom the organisation relies. ${ }^{25}$ One situation where it seems to be more effective is where organisations are in such crisis that accepting the painful therapy of re-engineering is the only way to survive.

Evaluations of two re-engineering pilots within the NHS suggest that personal commitment from the Chief Executive and other senior managers was an important factor in making the improvements which were achieved, but clinical resistance to what was seen as a brutal and inappropriate technique proved to be a major obstacle. ${ }^{21}{ }^{24}$ In both sites re-engineering had to be adapted to a more incremental and negotiated style to secure the involvement of clinicians. While this reflects the reality of managing change in health care, it implies a withdrawal from the grander ambition of transformational change; in practice, re-engineering has come much closer to TQM/CQI than the clear theoretical differences between these approaches would suggest: 
"Second order change rhetoric gave way to first order impact."21 (page 272)

"If BPR has to be applied incrementally and selectively, it doesn't look so very different from other quality initiatives such as TQM." ${ }^{24}$ (pp 413-4)

\section{REDESIGN: BLENDING QUALITY IMPROVEMENT APPROACHES}

A direct link exists between the re-engineering pilots and current redesign initiatives in the UK; a number of key players from the re-engineering pilot sites now occupy senior roles in the Modernisation Agency and the NHS Executive and their thinking has influenced the NHS Plan. ${ }^{7}$ In the US the Institute for Healthcare Improvement is grounded more in the TQM/CQI tradition, which has consistently been advocated by its director, Don Berwick. ${ }^{1326}$ However, both agencies-and others around the world-are conscious that the evidence suggests both approaches have major limitations and have not been as successful as was hoped.

Their thinking is converging on redesign as an attempt to retain and combine the best elements of re-engineering and TQM/CQI but avoiding the pitfalls. In particular, these agencies are experimenting with ways to retain the benefits of gradual negotiated improvement without losing the organisation-wide lateral vision of re-engineering and the opportunity to make discontinuous revolutionary changes as well as continuous improvement. Although redesign is often presented by its advocates as a single new entity, it is in fact more of an umbrella term covering a range of approaches with varying degrees of emphasis and eclectic use of tools and frameworks. This range can be seen in the practical examples given in boxes 3,4, and 5. The first example shows all the hallmarks of a classic CQI process, but with some more substantial changes in role with the introduction of a pharmacy technician. The second example has more in common with the radical questioning of traditional assumptions and simultaneous removal of several steps in the process associated with re-engineering. In the third example the use of continuous "Plan-Do-Study-Act" (PDSA) cycles is combined with the ambitious transformational aim of rapid improvement across organisational boundaries beyond the usual reach of individual work teams.

\section{THE CHALLENGE FOR REDESIGN}

Deciding how to combine apparently opposing philosophies is a difficult task; if redesign is to fare better than its predecessors it has to manage a number of balancing acts between apparent "dualities". ${ }^{27}$ A number of tensions can be identified:

(1) Change will not work unless it is owned and led by clinical teams. This requires a gradual negotiated approach as advocated by TQM/CQI, which will be enhanced by support from clinical opinion leaders. Although TQM/CQI has had more success in gaining clinical ownership than reengineering, clinicians have been sceptical about the sweeping claims of many quality improvement techniques imported from the private sector and have used their professional autonomy to resist. Equally, the evidence suggests that strong senior leadership (clinical and managerial) is needed to support front line teams, remove obstacles, and negotiate horizontal cross-boundary relationships if quality improvement is to have any deep and lasting effect.

(2) Change has to be manageable: testing incremental improvements is a safe way to learn about redesign and foster a more receptive culture. But redesign also needs lateral thinking which looks beyond existing processes and addresses whole systems, otherwise incremental improvements are likely to remain small scale with little impact on the wider organisation. Here, the "blank sheet" approach of reengineering which challenges traditional assumptions has much to offer. Again, senior leadership is likely to be key to keeping an eye on the big picture and ensuring smaller improvements are consistent with the overall direction, but it is important that this is top level vision and navigation rather than top down imposition. Trying to retain this radical vision is perhaps one of the most serious challenges for redesign; at present, many initiatives described as redesign do not in fact look very different from simple CQI projects.

(3) Early successes help gain interest and acceptanceinnovations are more likely to succeed when people can see demonstrable benefits. ${ }^{28}$ However, policy makers need to recognise that ambitions for radical overnight transformation are unrealistic and that change takes time:

- Clinical and managerial staff need dedicated time set aside.

- Individuals and organisations need time to learn, to reflect, and to implement.

- Cultural change-to a new way of thinking-is a long evolutionary process.

(4) Redesign must be demystified: much of it is common sense, accessible to all, and not the preserve of a few initiates. The tendency for each new quality improvement theory to generate its own jargon and esoteric knowledge must be resisted if clinicians are to become involved effectively. But the change in thinking and the facilitation skills needed require a major investment in training and development. Developing enough people of the right calibre at all levels will be a major task. If redesign is to be a primary vehicle for change, development needs to encompass top level leaders, not just isolated change teams working against the current. A particular challenge will be to ensure that training stresses the need to engage and learn from patients themselves at all stages of redesign.

Perhaps one of the most difficult challenges facing redesign is managing the weight of political expectation. As Walshe and Freeman ${ }^{29}$ have recently argued, the evidence suggests that most quality improvement interventions work some of the time, but rarely live up to the dramatic claims made for them in their early stages. Success is contingent upon multiple factors, including the manner of implementation in each setting and specific local contextual factors. Redesign faces the same issues of achieving change in a highly complex professionalised organisation as other quality improvement techniques. Although it seeks to learn from past evidence, there can be no guarantee that it will fare dramatically better. Indeed, emerging findings on the first wave of some UK redesign initiatives suggest a similar pattern of quality improvements being achieved but not being as extensive as hoped. ${ }^{30-32}$

Walshe and Freeman ${ }^{29}$ argue for careful research into quality improvement initiatives, but suggest that research which looks only at whether they work will probably just reiterate the findings that their impact is very variable and their effectiveness mixed. Research which examines how and why they work in some cases is likely to be more productive, and it is important that such research should influence the further development of redesign initiatives. However, redesign faces the very real danger

Key messages

- Redesigning health care has become an international preoccupation.

- Redesign draws on a number of quality improvement approaches, notably TQM/CQI and re-engineering.

- It seeks to learn from past experience of both approaches, and to balance the more gradual negotiated approach of TQM/CQI with the organisation-wide lateral thinking of re-engineering.

- Redesign has generated high political expectations, but faces the same issues of achieving change in a complex professionalised organisation as other quality improvement techniques. 
that its success in convincing politicians it can turn health care around will be followed by disappointment and rejection when the gains prove to be more modest than expected.

\section{REFERENCES}

1 Smith J. Redesigning health care: radical redesign is a way to radically improve. BM 2001;322:1257-8.

2 http://www.ihi.org.

3 http://www.modern.nhs.uk.

4 http://www.cbo.nl/english/cbo.htm

5 http://www.safetyandquality.org.

6 Heclo H. Modern social politics in Britain and Sweden. New Haven: Yale University Press, 1974

7 Department of Health. The NHS Plan. A plan for investment, a plan for reform. London: Department of Health, 2000.

8 Morrison I, Smith R. Hamster health care: time to stop running faster and redesign health care. BN 2000;321:1541-2.

9 Goldratt EM, Cox J. The goal. 2nd ed. Aldershot: Gower, 1993.

10 Womack J, Jones D. From lean production to the lean enterprise. Harvard Business Rev 1994: 93-104.

11 Plsek P, Wilson T. Complexity, leadership and management in healthcare organisations. BM 2001;323:746-9.

12 Deming WE. Out of the crisis: quality productivity and the competitive position. Cambridge, Mass.: MIT Press, 1986

13 Berwick D. Sounding board: Continuous improvement as an ideal in health care. N Engl J Med 1989;320:53-6.

14 Batalden PB, Stoltz PK. A framework for the continual improvement of health care: building and applying professional and improvement knowledge to test changes in daily work. Jt Comm J Qual Improve 1993; 19:424-51.

15 Hammer M, Champy J. Reengineering the corporation: a manifesto for business transformation. New York: Brealey, 1993.

16 Harrison S, Hunter D, Marnoch G, et al. Just managing: power and culture in the NHS. Basingstoke: Macmillan, 1992.

17 Joss R, Kogan M. Advancing quality: TQM in the NHS. Buckingham: Open University Press, 1995.

18 Shortell S, Bennet C, Byck G. Assessing the impact of continuous quality improvement on clinical practice: what will it take to accelerate progress? Milbank Q 1998:76:593-624.

19 Shortell SM, Jones RH, Rademaker AW, et al. Assessing the impact of total quality management and organizational culture on multiple outcomes of care for coronary artery bypass graft surgery patients. Med Care 2000;38:207-17.

20 Pollitt C. Business approaches to quality improvement: why are they hard for the NHS to swallow? Qual Health Care 1996;5:104-10.
21 McNulty T, Ferlie E. Reengineering health care: the complexities of organisational transformation. Oxford: Oxford University Press, 2002 22 Buchanan D. The limitations and opportunities of BPR in a politicised organisational climate. Human Relations 1997;50:51-72.

23 Walston K, Burns LR, Kimberley JR. Does reengineering really work? An examination of the context and outcomes of hospital reengineering initiatives. Admin Sci Q 2000, 42:366-94.

24 Packwood T, Pollitt C, Roberts S. Good medicine? A case study of business process reengineering in a hospital. Policy and Politics 1998;26:401-15.

25 Grint K, Case P. The violent rhetoric of reengineering: management consultancy on the offensive. J Manag Stud 1998;35:557-77

26 Berwick DM. Developing and testing changes in delivery of care. Ann Intern Med 1998;128:651-6.

27 Pettigrew A, Fenton E. The innovating organization. London: Sage, 2000.

28 Rogers E. The diffusion of innovations. 4th ed. New York: Free Press, 1995

29 Walshe K, Freeman T. Effectiveness of quality improvement: learning from evaluations. Qual Saf Health Care 2002;11:85-7.

30 Ham C, Kipping R, McLeod H, et al. Capacity, culture and leadership: lessons from experience of improving access to hospital services. Birmingham: Health Services Management Centre, University of Birmingham, 2002

31 Parker H, Meredith P, Kipping R, et al. Improving patient experience and outcomes in cancer: the early phase of the NHS Cancer Services Collaborative. Birmingham: Health Services Management Centre, University of Birmingham, 2001.

32 Bate SP, Robert G, Mcleod H. Report on the 'breakthrough collaborative approach to quality and service improvement within four regions of the NHS. A research based investigation of the Orthopaedic Services Collaborative within the Eastern, South \& West, South East and Trent regions. Birmingham: Health Services Management Centre, University of Birmingham, 2002.

33 Institute for Healthcare Improvement. Newsletter: Continuous improvement. Issue 12, March 2002 (available at http://www.ihi.org/ resources/successstories/ci0302mcoc.asp).

34 Stevenson B, Neal P. Eye opener. Health Service J 11 May 2000: 28-9.

35 Institute for Healthcare Improvement. Further information available at http://www.ihi.org/collaboratives.

36 Langley G, Nolan K, Nolan T, et al. The improvement guide: a practical approach to enhancing organizational performance. San Francisco, CA Jossey-Bass Publishers, 1996

37 Kerr D, Bevan H, Gowland B, et al. Redesigning cancer care. BM 2002;324:164-6.

Correction

Heroes and Martyrs of Quality and Safety

In the article entitled "John Williamson and the terrifying results of the Medical Practice Information Demonstration Project" by Duncan Neuhauser which appeared in the December 2002 issue of the journal (Qual Saf Health Care $2002 ; 11: 387-9)$, in paragraph 5 of the second column on page 388 the Director of the National Library of Medicine was incorrectly given as $\mathrm{Dr}$ Donald Lundberg. This should have been Dr Donald Lindberg. 